\title{
S
}

\section{Highly Conductive Molecular Junctions Based on Direct Binding of Benzene to Platinum Electrodes}

\author{
M. Kiguchi, ${ }^{1, *}$ O. Tal, ${ }^{1}$ S. Wohlthat, ${ }^{2,3}$ F. Pauly, ${ }^{3}$ M. Krieger, ${ }^{1, \dagger}$ D. Djukic, ${ }^{1}$ J. C. Cuevas, ${ }^{4,3}$ and J. M. van Ruitenbeek ${ }^{1}$ \\ ${ }^{1}$ Kamerlingh Onnes Laboratory, Leiden University, Leiden, The Netherlands \\ ${ }^{2}$ School of Chemistry, The University of Sydney, Sydney, Australia \\ ${ }^{3}$ Institut für Theoretische Festkörperphysik and DFG-Center for Functional Nanostructures, Universität Karlsruhe, \\ Karlsruhe, Germany \\ ${ }^{4}$ Departamento de Física Teórica de la Materia Condensada, Universidad Autónoma de Madrid, Madrid, Spain
}

(Received 5 March 2008; published 21 July 2008)

\begin{abstract}
Highly conductive molecular junctions were formed by direct binding of benzene molecules between two Pt electrodes. Measurements of conductance, isotopic shift in inelastic spectroscopy, and shot noise compared with calculations provide indications for a stable molecular junction where the benzene molecule is preserved intact and bonded to the Pt leads via carbon atoms. The junction has a conductance comparable to that for metallic atomic junctions (around $0.1-1 G_{0}$ ), where the conductance and the number of transmission channels are controlled by the molecule's orientation at different interelectrode distances.
\end{abstract}

PACS numbers: 73.63.Rt, 31.10.+z, 73.40.-c

Connecting a molecule as a bridge between two conducting electrodes is one of the fundamental challenges involved with the study of electron transport through molecular junctions [1]. The difficulty stems from a variety of requirements which are sometimes contradictory: a good molecule-electrode contact should be easy to achieve, for example, by chemical binding, but chemically insensitive to environmental influences, mechanically stable but flexible enough to allow molecular rearrangement, provide a good electronic coupling between the molecule and the conducting electrodes but still preserve to some degree the individual electronic properties of the molecule. A common approach in fabrication of such molecular junctions utilizes functional side groups attached to the main molecule structure as anchoring "arms" that chemically bind to metallic leads (e.g., thiol [2], amine [3], and carboxylic [4] groups). The thiol group became the most widely used anchoring group [2,5-7], since it binds readily to gold electrodes with some flexibility for rearrangement while providing a finite electronic coupling between the molecule and the leads. However, the use of thiols and other anchoring side groups poses some inherent disadvantages: the measured conductance of thiol based molecular junctions is distributed over a wide range of values [5-7] which is mainly ascribed to different binding configurations on $\mathrm{Au}$ electrodes [7-9]. Although other anchoring groups may overcome this drawback [3], in general, the anchoring groups act as resistive spacers between the electrodes and the molecule. This leads to low conductivity and sensitivity to different environmental effects such as neighbor adsorbed species [10].

Here, we report on a highly conductive molecular junction achieved by direct binding of a $\pi$-conjugated organic molecule (benzene) to metallic electrodes $(\mathrm{Pt})$ without the use of anchoring groups. The formation of such a molecular junction is verified by conductance measurements as well as the effect of isotopic substitution on inelastic electron spectroscopy. The evolution of conductance, vibration modes, and transmission channels as a function of the interelectrode distance is studied by comparing measurements of conductance, inelastic electron spectroscopy, and shot noise to theoretical calculations.

The measurements were performed using mechanically controllable break junctions [11]. A notched Pt wire (0.1 mm diameter, $99.99 \%$ purity) was glued on top of a bending beam and mounted in a three-point bending configuration inside a vacuum chamber. Once under vacuum and cooled to $4 \mathrm{~K}$, the wire was broken by mechanical bending of the substrate. The bending can be relaxed to form an atomic-sized junction between the Pt wire ends using a piezo element for fine adjustment. The formation of a clean Pt junction is verified by conductance histograms made from more than 3000 conductance traces taken during repeated junction stretching, as presented in Fig. 1 (black curve). The single peak around $1.5 G_{0}\left(G_{0}=\right.$ $2 e^{2} / h$ is the conductance quantum) is the typical signature of a clean Pt junction [12].

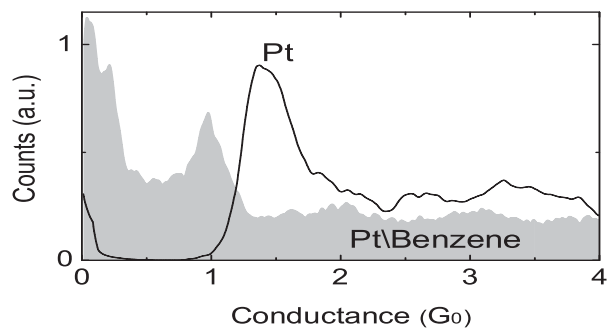

FIG. 1. Conductance histograms (normalized to the area under the curves) for a Pt junction (black), and for Pt after introducing benzene (filled). Each conductance histogram is constructed from more than 3000 conductance traces recorded with a bias of $0.1 \mathrm{~V}$ during repeated breaking of the contact. 
Benzene (Sigma-Aldrich, purity $\geq 99.9 \%$ ) was degassed through repeated freeze-pump-thaw cycles. Following the formation of the Pt junction, the benzene was admitted using a leak valve via a heated capillary to the Pt junction while the latter is broken and formed repeatedly. During the benzene introduction, the typical Pt peak is observed to be suppressed, and a single peak appears near $1 G_{0}$ accompanied with a low conductance tail (Fig. 1, filled curve). In some cases, the histogram exhibits a peak near $0.2 G_{0}$ on top of the tail. These findings imply that after the introduction of benzene, the formation of pure $\mathrm{Pt}$ junctions is suppressed while new junctions with preferred conductance of $1 G_{0}$ and sometimes $0.2 G_{0}$ are formed while stretching the contact.

Differential conductance $(d I / d V)[13]$ was measured as a function of voltage across the $\mathrm{Pt} /$ benzene junctions where each curve was recorded at fixed electrodes separation. As demonstrated at the top of Fig. 2(a), a symmetric upward step in the differential conductance is observed at $40 \mathrm{mV}$ [as can be determined more accurately by the corresponding peaks in the derivative $\left(d^{2} I / d V^{2}\right)$ in the lower panel of Fig. 2(a)]. The step feature indicates on a vibration which is excited by the transport electrons having an energy of $40 \mathrm{meV}$ [12]. The conductance enhancement is typical for inelastic electron tunneling spectroscopy, as expected for vibration excitation at zero bias conductance below $0.5 G_{0}[14,15]$. In order to accurately determine the associated vibration energy, 250 differential conductance spectra were collected for junctions having a zero bias conductance of $0.05-0.4 G_{0}$, resulting in the energy histogram presented in Fig. 2(b) (circles). The histogram shows a well-defined peak at $42 \mathrm{meV}$ with $5 \mathrm{meV}$ width. The vibration energy for a given junction is insensitive to junction stretching, unlike the vibration modes for $\mathrm{Pt} / \mathrm{H}_{2}$ junctions [12]. We also measured the differential conductance spectra for junctions with a conductance close to $1 G_{0}$, but the positions in energy of the conductance steps do not reveal any well-defined vibration energies, in con-
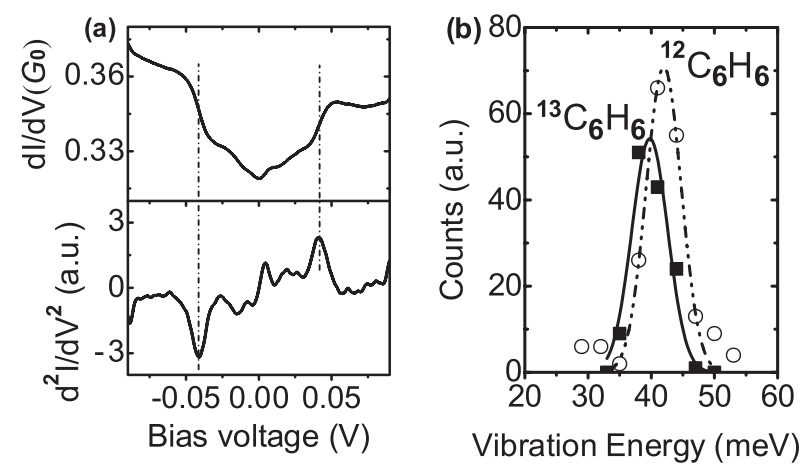

FIG. 2. (a) Differential conductance (top) and its derivative (bottom) for Pt contact after introduction of benzene taken at a zero bias conductance of $0.3 G_{0}$. (b) Distribution of vibration energy at low conductance regime. trast to the junctions having a conductance below $0.4 G_{0}$ [Fig. 2(b)].

We repeated the experiment using ${ }^{13} \mathrm{C}$ isotope substitution in all six positions of benzene (Buchem, $99 \%{ }^{13} \mathrm{C}_{6} \mathrm{H}_{6}$, purity $\geq 98 \%$ ). The mass ratio of ${ }^{13} \mathrm{C}_{6} \mathrm{H}_{6}$ and ${ }^{12} \mathrm{C}_{6} \mathrm{H}_{6}$ is $84 / 78$, from which we predict a shift of the vibration mode from $42 \mathrm{meV}$ by the square root of this ratio to $40 \mathrm{meV}$ for a vibration mode that involves the movement of the whole molecule. The $\mathrm{Pt} /{ }^{13} \mathrm{C}_{6} \mathrm{H}_{6}$ energy histogram obtained from 130 differential conductance spectra [filled squares in Fig. 2(b)] shows a peak at $40 \mathrm{meV}$ with $5 \mathrm{meV}$ width, in excellent agreement with the shift expected for a molecular vibration mode which involves the movement of the entire benzene molecule. This observation supports the picture of transport electrons crossing a junction composed from a benzene molecule.

Focusing on the way that electrons are transmitted through the junction, we turn to shot noise measurements. As the shot noise level is determined by the number of available transmission channels across the junction and their transmission probabilities, $\tau_{i}$, the main transmission probabilities can be resolved by comparing noise and conductance measurements to the expression for noise power for an arbitrary set of transmission probabilities, at temperature $T$ and bias voltage $V$ as given by [16]

$$
S_{I}=2 e V \operatorname{coth}\left(\frac{e V}{2 k T}\right) \frac{2 e^{2}}{h} \sum_{i} \tau_{i}\left(1-\tau_{i}\right)+4 k T \frac{2 e^{2}}{h} \sum_{i} \tau_{i}^{2}
$$

where $k$ is Boltzmann's constant. Using the method described in Refs. [15,17], we have measured the shot noise [i.e., $S_{I}(V>0)-S_{I}(V=0)$ ] across junctions with different conductance values. Once a stable junction was established at a certain conductance, the noise power was measured as a function of frequency at different bias currents. At each bias, 10000 noise spectra where averaged. Differential conductance spectra were measured before and after every set of noise measurements to verify that the same contact was maintained during the measurements.

Figure 3 presents three sets of shot noise measurements as a function of bias current. For junctions with zero bias conductance of 1.08 (squares), 0.71 (open triangles), and $0.20 \pm 0.01 G_{0}$ (bullets), the following transmission probabilities were obtained: $\{0.68,0.40\},\{0.36,0.25,0.10\}$, and $\{0.20\}$, respectively. The number of channels is eventually reduced to one when the conductance is reduced to $0.2 G_{0}$, while at higher conductance (also well below $1 G_{0}$ ), multiple channels make up the transport across the junction. As opposed to $\mathrm{Pt} / \mathrm{H}_{2} \mathrm{O}$ junctions, there is no dominant transmission channel when more than a single channel exists [15]. Note that the uncertainty in the value of the main transmission probabilities are small (in this case \pm 0.01 ), since the fit is very sensitive to the number of channels and their probabilities $[17,18]$. Choosing more channels than 


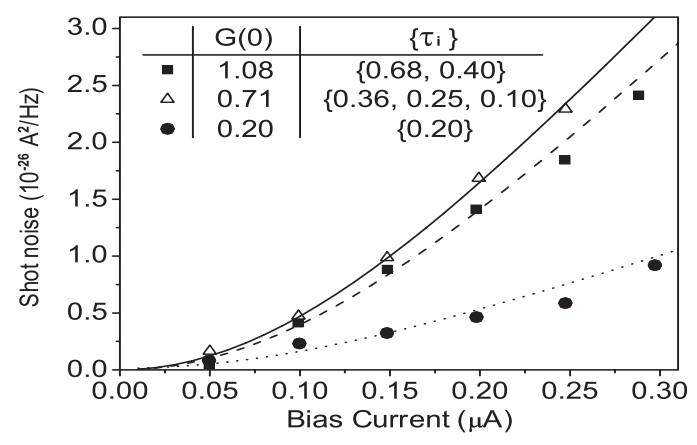

FIG. 3. Shot noise as a function of the bias current across the $\mathrm{Pt}$ /benzene junction. The zero bias conductance $[G(0)]$ for this junction is $1.08 G_{0}$ (filled squares), $0.71 G_{0}$ (hollow triangles), and $0.2 G_{0}$ (filled circles). Fitting the data with theory (curves) gives the decomposition of the total transmission in terms of transmission probabilities, $\tau_{i}$, of the conduction channels (where $\left.G=\sum_{i} \tau_{i} G_{0}\right)$ as shown in the inset.

presented in Fig. 3 (inset) is restricted to small additional channels that do not affect the specified probabilities (within \pm 0.01 ).

In order to better understand the experimental observations in terms of a Pt/benzene junction, we have performed extensive DFT calculations using TURBOMOLE v5.7 [19], where we have used a split valence polarization basis set for all nonhydrogen atoms [20] and the BP86 exchangecorrelation functional [21]. To determine the possible geometries of the molecular junction and its evolution upon stretching, we proceed as follows. We first place a benzene molecule in between two Pt clusters composed by 8 atoms sitting in the sites of an fcc lattice, which simulate two atomically sharp electrode tips oriented in the (111) direction with a lattice parameter of $3.92 \AA$. Then, the geometry is optimized holding the Pt electrodes fixed. Finally, we move apart the pyramids stepwise and relax the molecule in each step.

Although other junction geometries are possible, we focused on the analysis of the junction evolution during stretching shown at the top of Fig. 4, which seems to be compatible with many of the experimental observations described above. Initially, the benzene sits on top of the Pt electrodes with its plane perpendicular to the $\mathrm{Pt}$ junction axis and bonded to both pyramids with two $\mathrm{C}$ atoms. At an interelectrodes distance of $4.5 \AA$, the molecule jumps to a central position between the electrodes to form a molecular junction. During the junction stretching, the molecule is progressively tilted while the number of $\mathrm{C}$ atoms that bind to each Pt electrode [22] is reduced from three to two and eventually to one. Notice that during the junction evolution, the benzene retains its molecular integrity (in particular, there is no dehydrogenation). The total energy has a minimum shortly after the formation of the benzene bridge [Fig. 4(a), dots], which indicates the most stable configuration.

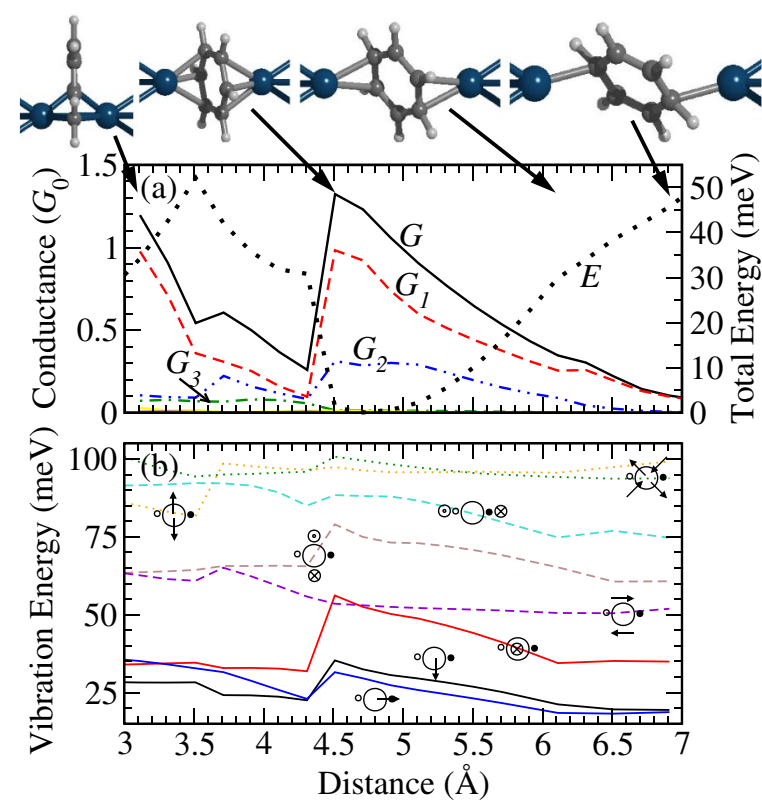

FIG. 4 (color online). Simulation of the stretching process of a $\mathrm{Pt} /$ benzene junction. (a) Total conductance, $G$, the contribution of the individual conduction channels, $G_{i}=G_{0} \tau_{i}$, and the change of the total energy, $E$, as a function of the distance between the Pt tip atoms. (b) Stretching dependence of the energy of the benzene vibration modes in the range between 0 and $105 \mathrm{meV}$. The translation modes are plotted as solid lines, the rotation modes as dashed lines and the stretch modes as dotted lines. The character of the modes is indicated by symbols, where benzene is represented by a large circle, the Pt atoms by two small circles and arrows depicted the motion of the molecule.

For each geometry, we have computed the vibration modes of the junction [Fig. 4(b)], keeping all Pt atoms fixed, which is justified by the large difference in mass between $\mathrm{C}$ or $\mathrm{H}$ and $\mathrm{Pt}$. At electrode separations larger than $4.5 \AA$, there are two possible candidates for the mode observed in the experiment. The first one is a longitudinal mode, where the entire molecule oscillates back and forth along the junction axis between the electrodes. This mode becomes softer as the junction is elongated leading to energy decrease from 50 to $35 \mathrm{meV}$ at large distances. The second mode involves a molecular rotation with respect to its $\mathrm{C}_{6}$-axis, having an energy around $50 \mathrm{meV}$. This energy is rather insensitive to elongation of the contact, involves the motion of the whole molecule, and has the appropriate energy in the appropriate conductance range (see below). For these reasons, this vibration is our main candidate for the mode observed in the experiment.

To calculate the conductance of these molecular junctions, we have used Green's function techniques and the Landauer formula expressed in a local nonorthogonal basis, as discussed in detail in Refs. [23,24]. We obtain the bulk Green's functions following the procedure described in Ref. [24] by computing separately the electronic struc- 
ture of a spherical Pt fcc cluster with 459 atoms. Additionally, we obtain from this analysis a value for the Fermi energy of $-5.4 \mathrm{eV}$.

In Fig. 4(a), we show the calculated results for the conductance and its decomposition into conduction channels. In the range where the benzene molecule sits on top of the Pt-Pt bond, the conductance decreases with stretching from a value above $1 G_{0}$ down to $0.2 G_{0}$, while the current is mainly carried by three channels. When the benzene moves in between the electrodes to form a molecular junction, the conductance jumps up to $1.3 G_{0}$. Then, when the total energy reaches its minimum (i.e., the most stable geometry), the conductance is very close to $1 G_{0}$, which may explain the main peak in the conductance histogram (Fig. 1). As the stretching proceeds, the conductance decreases monotonically, and the current is mainly controlled by two channels and finally by only one in the last stages. We find some similarity between the channel decomposition obtained by noise measurements and the calculated one at interelectrodes distances of 3.4, 4.9, and $6.6 \AA$ for the junctions with conductance of $0.71,1.08$, and $0.20 G_{0}$, respectively. In general, the calculated transmission probabilities indicate a lack of a dominant channel when more than a single channel exists and a reduction of the number of channels when the conductance of the molecular junction (for distances larger than $4.5 \AA$ ) is reduced as was also implied by the experimental findings. Interestingly, as a rule of thumb, an upper limit to the number of conduction channels when the molecular junction is formed, is simply given by the number of $\mathrm{C}$ atoms bonded to the $\mathrm{Pt}$ tip atoms. This can be understood as follows. Since each $\mathrm{C}$ atom has only one orbital taking part in the $\pi$-system of the benzene ring, each atom can build at most one $\pi$-channel. For distances smaller than $4.5 \AA$, channels involving $\sigma$-bonds might play a role, as well as direct tunneling from one $\mathrm{Pt}$ electrode to the other.

Our experimental observations together with the agreement in many points between the experimental results and the calculations indicate the formation of a stable and highly conductive junction by binding a benzene molecule to the platinum electrodes via Pt-C bonds while preserving its molecular structure. Stretching of the junction leads to tilting of the molecule which reduces both the conductance and the number of transmission channels across the junction as a consequence of sequential breaking of the Pt-C bonds.

This work is part of the research program of the "Stichting FOM," which is financially supported by NWO. O. T. is also grateful to IUVSTA-Welch Foundation for their support. M. K. greatly acknowledges the support by the European Commission (RTN DIENOW). S. W. acknowledges the Australian Partnership for Advanced Computing for computing resources, F. P. the support by the Young Investigator Group at KIT, and J. C. C. the financial support of the EU network BIMORE (No. MRTN-CT-2006-035859).

*Present address: Graduate School of Science, Hokkaido University, Sapporo, Japan, and JST-PRESTO

${ }^{\dagger}$ Present address: Institute of Applied Physics, University of Erlangen-Nürnberg, Erlangen, Germany

[1] N. J. Tao, Nature Nanotechnology 1, 173 (2006).

[2] M. A. Reed, C. Zhou, C. J. Muller, T. P. Burgin, and J. M. Tour, Science 278, 252 (1997).

[3] L. Venkataraman, J.E. Klare, I. W. Tam, C. Nuckolls, M.S. Hybertsen, and M.L. Steigerwald, Nano Lett. 6, 458 (2006).

[4] F. Chen, X. Li, J. Hihath, Z. Huang, and N. Tao, J. Am. Chem. Soc. 128, 15874 (2006).

[5] J. Ulrich, D. Esrail, W. Pontius, L. Venkataraman, D. Millar, and L.H. Doerrer, J. Phys. Chem. 110, 2462 (2006).

[6] X. Li, J. He, J. Hihath, B. Xu, S. M. Lindsay, and N. Tao, J. Am. Chem. Soc. 128, 2135 (2006).

[7] C. Li, I. Pobelov, T. Wandlowski, A. Bagrets, A. Arnold, and F. Evers, J. Am. Chem. Soc. 130, 318 (2008).

[8] M. Fujihira, M. Suzuki, S. Fujii, and A. Nishikawa, Phys. Chem. Chem. Phys. 8, 3876 (2006).

[9] K. H. Müller, Phys. Rev. B 73, 045403 (2006).

[10] D. P. Long, J. L. Lazorcik, B. A. Mamtooth, M. H. Moore, M. A. Ratner, A. Troisi, Y. Yao, J. W. Ciszek, J. M. Tour, and R. Shashidhar, Nat. Mater. 5, 901 (2006).

[11] C. J. Muller, J. M. van Ruitenbeek, and L. J. de Jongh, Physica C (Amsterdam) 191, 485 (1992).

[12] D. Djukic, K. S. Thygesen, C. Untiedt, R. H. M. Smit, K. W. Jacobsen, and J. M. van Ruitenbeek, Phys. Rev. B 71, 161402(R) (2005).

[13] R. H. M. Smit, Y. Noat, C. Untiedt, N. D. Lang, M. C. van Hemert, and J. M. van Ruitenbeek, Nature (London) 419, 906 (2002).

[14] M. Paulsson, T. Frederiksen, and M. Brandbyge, Phys. Rev. B 72, 201101(R) (2005).

[15] O. Tal, M. Krieger, B. Leerink, and J. M. van Ruitenbeek, Phys. Rev. Lett. 100, 196804 (2008).

[16] Y. M. Blanter and M. Büttiker, Phys. Rep. 336, 1 (2000).

[17] D. Djukic and J. M. van Ruitenbeek, Nano Lett. 6, 789 (2006).

[18] H. E. van den Brom and J. M. van Ruitenbeek, Phys. Rev. Lett. 82, 1526 (1999).

[19] R. Ahlrichs, M. Bär, M. Häser, H. Horn, and C. Kölmel, Chem. Phys. Lett. 162, 165 (1989).

[20] A. Schäfer, H. Horn, and R. Ahlrichs, J. Chem. Phys. 97, 2571 (1992).

[21] J. P. Perdew, Phys. Rev. B 33, 8822 (1986).

[22] We consider that a $\mathrm{C}$ atom is coupled to the Pt electrodes if the Pt-C bond distance is smaller than $2.65 \AA$.

[23] S. Wohlthat, F. Pauly, J.K. Viljas, J.C. Cuevas, and G. Schön, Phys. Rev. B 76, 075413 (2007).

[24] F. Pauly, Ph.D. thesis, Universität Karlsruhe, 2007. 